\title{
The effects of large vibration amplitudes on the mode shapes and natural frequencies of thin isotropic skew plates
}

\author{
H. Mly Abdelali ${ }^{1}$, K. El Bikri ${ }^{2}$ and R. Benamar ${ }^{1}$ \\ ${ }^{1}$ EMI, EGT, LERSIM, Agdal University Mohammed V-Souissi, Rabat, Morocco \\ ${ }^{2}$ ENSET-Rabat, Mechanical Engineering Department, LaMIPI, University Mohammed V-Souissi, Rabat, Morocco
}

\begin{abstract}
The present work concerns the nonlinear dynamic behavior of skew plates at large vibration amplitudes with fully clamped edges. The large amplitude free vibration problem is modelled by a set of nonlinear algebraic equations using Hamilton's principle and spectral analysis. The natural frequencies and modes of vibration have been obtained for fully clamped edges for various values of the skew angle. The relationship between the amplitude and frequencies is studied for isotropic skew plates. It was found that the frequencies of non linear vibration increase with increasing amplitude. Numerical details are presented and results are given, corresponding to the linear case and to the nonlinear case for various values of $\theta$ and various amplitudes of vibration. The results, compared to the previous ones available in the literature, show a reasonably good agreement.
\end{abstract}

\section{Introduction}

In contemporary structures, skew plates are used in diverse domains, such as stiffened plate, used as floors in bridges, ship hulls, buildings, etc. The simulation of static and dynamic behavior of skew plates is an interesting area of work for researchers. Alwar and Rao (1973) [5] presented a nonlinear analysis of orthotropic skew plates of constant thickness subjected to a uniform transverse load. G. Prathap and T. K. Varadan (1978) [4] studied the large amplitude free flexural vibrations of thin, elastic anisotropic skew plates. Chia (1980) [6] considered the moderately large deflection elastic behaviour of homogenous isotropic and laminated anisotropic rectangular as well as skew plates by analytical methods. Xiang et al. (1995) [7] studied the elastic buckling behaviour of skew Mindlin plate under a shear load. M.K. Singha and Rupesh Daripa (2007) [8] investigate the large amplitude free flexural vibration behavior of symmetrically laminated composite skew plates.

In the present study, we apply the theoretical model developed in [1] to analyze the geometrically nonlinear free dynamic response of an isotropic fully clamped skew plate in order to investigate the effect of nonlinearity on the nonlinear resonance frequency at large vibration amplitudes.

In the present paper, the general formulation of the model for nonlinear vibration of isotropic skew plates at large vibration amplitudes is presented first, and the relationships between the nonlinear resonance frequency ratio and the vibration amplitudes is given for values of the angle $\theta$ varying from $0^{\circ}$ to $45^{\circ}$. While there are many papers dealing with rectangular plate, as listed in reference, only few papers exist that deal with the large amplitude free flexural vibration of skew plates [4]. In reference [4], the authors obtained results for the large amplitude free flexural vibrations of thin, elastic anisotropic skew plates, on specializing for an isotropic skew plate and an orthotropic rectangular plate by using the von Karman field equations and the Berger approximation to study a skew plate.

\section{Theoretical formulations}

A skew plate with a skew angle $\theta$ is shown in Figure 1 . For the large displacement formulation, it is assumed that the material of the plate is elastic, isotropic and homogeneous. The thickness of the plate is considered to be sufficiently small so as to avoid the effect of shear deformation. Also the stress and strain measures are based on the original dimensions of the plate.

This is an Open Access article distributed under the terms of the Creative Commons Attribution License 2.0, which permits unrestricted use, distribution, and reproduction in any medium, provided the original work is properly cited. 


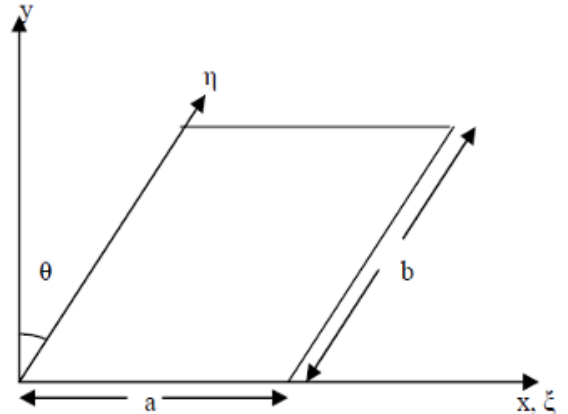

Fig. 1. Skew plate in $x-y$ and $\xi-\eta$ co-ordinate system.

Consider the transverse vibration of the skew plate shown in figure 1 having the following characteristics: $a, b, S$ : length, width and area of the plate; $x-y$ : plate co-ordinates in the length and the width directions; $\xi-\eta, H$ : Skew plate co-ordinate and thickness of the plate; E, $v$ : Young's modulus and Poisson's ratio; $D, \rho$ : bending stiffness of the plate and mass per unit volume of the plate.

The theoretical formulation of the skew plate large vibration amplitude problem is presented, which leads to a numerical model. By assuming harmonic motion and expanding the transverse displacement in the form of finite series of functions, the bending strain energy $V_{b}$, the axial strain energy $V_{a}$ and the kinetic energy $T$ are expressed. By discretization of the expressions for $V_{a}, V_{b}$ and $T$, the tensors $m_{i j}, k_{i j}$ and $b_{i j k l}$ are defined. The frequency equation at large vibration amplitudes is developed. Finally, the non dimensional formulation of the problem and details of the numerical solution are presented.

\subsection{Expression for the bending strain, axial and kinetic energies in the skew plate}

The potential energies due to bending and axial strains and the kinetic energy are given in $\mathrm{x}-\mathrm{y}$ co-ordinate by:

$$
\begin{aligned}
& V_{b}=\frac{D}{2} \int_{S}\left[\left(\frac{\partial^{2} w}{\partial x^{2}}+\frac{\partial^{2} w}{\partial y^{2}}\right)^{2}+2(1-v)\left(\left(\frac{\partial^{2} w}{\partial x \partial y}\right)^{2}-\right.\right. \\
& \left.\left.\left(\frac{\partial^{2} w}{\partial x^{2}} \frac{\partial^{2} w}{\partial y^{2}}\right)\right)\right] d S \\
& V_{a}=\frac{3 D}{2 H^{2}} \int_{S}\left[\left(\left(\frac{\partial w}{\partial x}\right)^{2}+\left(\frac{\partial w}{\partial y}\right)^{2}\right)^{2}\right] d S \\
& T=\frac{\rho H}{2} \int_{S}\left(\frac{\partial w}{\partial t}\right)^{2} d S
\end{aligned}
$$

The skew co-ordinates are related to the rectangular coordinate by: $\xi=x-y \tan \theta ; \eta=y / \cos \theta$.

The potential energies due to the bending and axial strains and the kinetic energy are given in the $\xi-\eta$ coordinate system by:

$$
\begin{aligned}
& V_{b}=\frac{D}{2} \int_{\mathrm{A}}\left[\frac{1}{\cos ^{4} \theta}\left(\frac{\partial^{2} w}{\partial \xi^{2}}+\frac{\partial^{2} w}{\partial \eta^{2}}-2 \sin \theta \frac{\partial^{2} w}{\partial \xi \partial \eta}\right)^{2}+\right. \\
& \left.\frac{2(1-v)}{\cos ^{2} \theta}\left(\left(\frac{\partial^{2} w}{\partial \xi \partial \eta}\right)^{2}-\left(\frac{\partial^{2} w}{\partial \xi^{2}} \frac{\partial^{2} w}{\partial \eta^{2}}\right)\right)\right] d A \\
& V_{a}=\frac{3 D}{2 H^{2}} \int_{A}\left[\frac{1}{\cos ^{4} \theta}\left(\left(\frac{\partial w}{\partial \xi}\right)^{2}+\left(\frac{\partial w}{\partial \eta}\right)^{2}-2 \sin \theta \frac{\partial^{2} w}{\partial \xi \partial \eta}\right)^{2}\right] d A \\
& T=\frac{\rho H}{2} \int_{\mathrm{A}}\left(\frac{\partial w}{\partial t}\right)^{2} d A
\end{aligned}
$$

where: $d A=\cos \theta d \xi d \eta$ and $W(\xi, \eta, t)$ is the transverse displacement function.

The plate functions used in the discretisation process are obtained as product of beam functions. The transverse displacement can be written as:

$w(\xi, \eta, t)=w(\xi, \eta) \sin \omega t$

where:

$w(\xi, \eta)=a_{i} w_{i}(\xi, \eta)$

The discretization and the non dimensional formulation is made as follows:

$k_{i j}^{*}=\int_{A^{*}}\left\{\alpha^{4} \frac{\partial^{2} w_{i}^{*}}{\partial \xi^{* 2}} \frac{\partial^{2} w_{j}^{*}}{\partial \xi^{* 2}}+\frac{\partial^{2} w_{i}^{*}}{\partial \eta^{* 2}} \frac{\partial^{2} w_{j}^{*}}{\partial \eta^{* 2}}+\alpha^{2} \frac{\partial^{2} w_{i}^{*}}{\partial \xi^{* 2}} \frac{\partial^{2} w_{j}^{*}}{\partial \eta^{* 2}}+\right.$

$\alpha^{2} \frac{\partial^{2} w_{i}^{*}}{\partial \eta^{* 2}} \frac{\partial^{2} w_{j}^{*}}{\partial \xi^{* 2}}-2 \sin \theta \alpha^{3} \frac{\partial^{2} w_{i}^{*}}{\partial \xi^{2}} \frac{\partial^{2} w_{j}^{*}}{\partial \xi^{*} \partial \eta^{*}}-2 \sin \theta \alpha \frac{\partial^{2} w_{i}^{*}}{\partial \eta^{*}} \frac{\partial^{2} w_{j}^{*}}{\partial \xi^{*} \partial \eta^{*}}-$

$2 \sin \theta \alpha^{3} \frac{\partial^{2} w_{i}^{*}}{\partial \xi^{*} \partial \eta^{*}} \frac{\partial^{2} w_{j}^{*}}{\partial \xi^{2}}-2 \sin \theta \alpha \frac{\partial^{2} w_{i}^{*}}{\partial \xi^{*} \partial \eta^{*}} \frac{\partial^{2} w_{j}^{*}}{\partial \eta^{* 2}}+$

$4 \sin ^{2} \theta \alpha^{2} \frac{\partial^{2} w_{i}^{*}}{\partial \xi^{*} \partial \eta^{*}} \frac{\partial^{2} w_{j}^{*}}{\partial \xi^{*} \partial \eta^{*}}+2(1-v) \cos ^{2} \theta \alpha^{2}\left[\left(\frac{\partial^{2} w_{i}^{*}}{\partial \xi^{*} \partial \eta^{*}} \frac{\partial^{2} w_{j}^{*}}{\partial \xi^{*} \partial \eta^{*}}\right)-\right.$

$\left.\left.\left(\frac{\partial^{2} w_{i}^{*}}{\partial \xi^{* 2}} \frac{\partial^{2} w_{j}^{*}}{\partial \eta^{* 2}}\right)\right]\right\} d \eta^{*} d \xi^{*}$

$b_{i j k l}^{*}=3 \int_{A^{*}}\left\{\alpha^{4} \frac{\partial w_{i}^{*}}{\partial \xi^{*}} \frac{\partial w_{j}^{*}}{\partial \xi^{*}} \frac{\partial w_{k}^{*}}{\partial \xi^{*}} \frac{\partial w_{l}^{*}}{\partial \xi^{*}}+\alpha^{2} \frac{\partial w_{i}^{*}}{\partial \xi^{*}} \frac{\partial w_{j}^{*}}{\partial \xi^{*}} \frac{\partial w_{k}^{*}}{\partial \eta^{*}} \frac{\partial w_{l}^{*}}{\partial \eta^{*}}-\right.$

$2 \sin \theta \alpha^{3} \frac{\partial w_{i}^{*}}{\partial \xi^{*}} \frac{\partial w_{j}^{*}}{\partial \xi^{*}} \frac{\partial w_{k}^{*}}{\partial \eta^{*}} \frac{\partial w_{l}^{*}}{\partial \xi^{*}}+\alpha^{2} \frac{\partial w_{i}^{*}}{\partial \eta^{*}} \frac{\partial w_{j}^{*}}{\partial \eta^{*}} \frac{\partial w_{k}^{*}}{\partial \xi^{*}} \frac{\partial w_{l}^{*}}{\partial \xi^{*}}+$

$\frac{\partial w_{i}^{*}}{\partial \eta^{*}} \frac{\partial w_{j}^{*}}{\partial \eta^{*}} \frac{\partial w_{k}^{*}}{\partial \eta^{*}} \frac{\partial w_{l}^{*}}{\partial \eta^{*}}-2 \sin \theta \alpha \frac{\partial w_{i}^{*}}{\partial \eta^{*}} \frac{\partial w_{j}^{*}}{\partial \eta^{*}} \frac{\partial w_{k}^{*}}{\partial \eta^{*}} \frac{\partial w_{l}^{*}}{\partial \xi^{*}}-$

$2 \sin \theta \alpha^{3} \frac{\partial w_{i}^{*}}{\partial \eta^{*}} \frac{\partial w_{j}^{*}}{\partial \xi^{*}} \frac{\partial w_{k}^{*}}{\partial \xi^{*}} \frac{\partial w_{l}^{*}}{\partial \xi^{*}}-2 \sin \theta \alpha \frac{\partial w_{i}^{*}}{\partial \eta^{*}} \frac{\partial w_{j}^{*}}{\partial \xi^{*}} \frac{\partial w_{k}^{*}}{\partial \eta^{*}} \frac{\partial w_{l}^{*}}{\partial \xi^{*}}+$

$\left.4 \sin ^{2} \theta \alpha^{2} \frac{\partial w_{i}^{*}}{\partial \eta^{*}} \frac{\partial w_{j}^{*}}{\partial \xi^{*}} \frac{\partial w_{k}^{*}}{\partial \eta^{*}} \frac{\partial w_{l}^{*}}{\partial \xi^{*}}\right\} d \eta^{*} d \xi^{*}$

$m_{i j}^{*}=\int_{\mathrm{A}^{*}} w_{i}^{*} w_{j}^{*} d \eta^{*} d \xi^{*}$

where:

$w_{i}(\xi, \eta)=H w_{i}^{*}\left(\frac{\xi}{a}, \frac{\eta}{b}\right)=H w_{i}^{*}\left(\xi^{*}, \eta^{*}\right)$

$\alpha=\frac{b}{a} \quad ; \xi^{*}=\frac{\xi}{a} \quad ; \eta^{*}=\frac{\eta}{b}$

The dynamic behavior of the isotropic skew plate is governed by Hamilton's principle which leads to the following set of non linear algebraic equations:

$2 a_{i} k_{i r}^{*}+3 a_{i} a_{j} a_{k} b_{i j k r}^{*}-2 \omega^{*} a_{i} m_{i r}^{*}=0, \quad \mathrm{r}=1 \ldots \mathrm{n}$ 
where:

$$
\begin{aligned}
\omega^{*} & =\frac{a_{i} a_{j} k_{i j}^{*}+a_{i} a_{j} a_{k} a_{l} b_{i j k l}^{*}}{a_{i} a_{j} m_{i j}^{*}} \\
\omega^{2} & =\frac{D}{\rho b^{4} \cos ^{4} \theta} \omega^{* 2}
\end{aligned}
$$

\section{Numerical details}

In the present work, calculations have been made using 9 plate functions obtained as product the $1^{\text {st }}$ three clampedclamped beam functions. and different values of $\theta\left(0^{\circ}\right.$, $15^{\circ}, 30^{\circ}$ and $45^{\circ}$ ).

Two types of validations have been performed:

1) The linear results for various values of $\theta$.

2) The non linear results obtained here and in previous works for various values of $\theta$.

The results and comparisons are summarized in Tables 1, 2 and 3. A satisfactory agreement can be noticed in spite of the little difference observed in the results, which may be due to use of the Berger approximation enforcing the immovable boundary condition in an averaged manner [4] whereas there is no such approximation involved in the present work.

Table 1. Nondimentional frequency parameters $\omega b^{2} \sqrt{\frac{\rho}{D}} \cos ^{2} \theta$

\begin{tabular}{|c|c|c|c|c|}
\hline \multicolumn{5}{|c|}{$\omega \mathrm{b}^{2} \sqrt{\frac{\rho}{\mathrm{D}}} \cos ^{2} \theta$ For values of skew angle, $\theta$, deg, of } \\
\hline & 0 & 15 & 30 & 45 \\
\hline Leissa [1] & 36.11 & 36.67 & 38.15 & 40.08 \\
\hline Present Work & 35.99 & 36.52 & 37.92 & 39.72 \\
\hline
\end{tabular}
for a C-C-C-C skew plate

Table 2. Ratio frequency parameters to the first nonlinear mode shape for a C-C-C-C square plate

\begin{tabular}{llll}
\hline W. Han and M. Petyt [3] & \multicolumn{2}{c}{ Present Work } \\
$w^{*_{\max }}$ & $\omega^{*_{n} /} / \omega^{*} 1$ & $w^{*_{\max }}$ & $\omega^{*_{n l} / \omega^{*}{ }_{l}}$ \\
\hline 0.20 & 1.010 & 0.20 & 1.010 \\
0.40 & 1.041 & 0.40 & 1.042 \\
0.60 & 1.089 & 0.60 & 1.090 \\
0.80 & 1.152 & 0.80 & 1.154 \\
1.00 & 1.226 & 1.00 & 1.227 \\
1.20 & 1.308 & 1.20 & 1.310 \\
1.40 & 1.397 & 1.40 & 1.396 \\
1.50 & 1.443 & 1.50 & 1.442 \\
\hline
\end{tabular}

Table 3. Ratio frequency parameters to the first nonlinear mode

\begin{tabular}{|c|c|c|c|c|c|c|}
\hline \multirow[t]{3}{*}{$w^{*}{ }_{\max }$} & \multicolumn{6}{|c|}{ Skew Angles } \\
\hline & \multicolumn{3}{|c|}{$\begin{array}{l}\text { G. Prathap and T. K. Varadan } \\
\text { [4] }\end{array}$} & \multicolumn{3}{|c|}{ Present Work } \\
\hline & $15^{\circ}$ & $30^{\circ}$ & $45^{\circ}$ & $15^{\circ}$ & $30^{\circ}$ & $45^{\circ}$ \\
\hline 0.2 & 1.009 & 1.008 & 1.007 & 1.010 & 1.010 & 1.010 \\
\hline 0.4 & 1.037 & 1.033 & 1.029 & 1.041 & 1.040 & 1.039 \\
\hline 0.6 & 1.082 & 1.073 & 1.065 & 1.089 & 1.086 & 1.089 \\
\hline 0.8 & 1.142 & 1.126 & 1.112 & 1.152 & 1.147 & 1.145 \\
\hline 1.0 & 1.214 & 1.191 & 1.171 & 1.224 & 1.220 & 1.214 \\
\hline 1.2 & 1.297 & 1.266 & 1.239 & 1.306 & 1.298 & 1.2917 \\
\hline 1.4 & 1.388 & 1.349 & 1.314 & 1.392 & 1.383 & 1.375 \\
\hline 1.5 & 1.437 & 1.393 & 1.355 & 1.437 & 1.427 & 1.419 \\
\hline
\end{tabular}
shape for a C-C-C-C skew plate

\section{Conclusion}

The theoretical model for thin elastic skew plate at large displacement has been developed and numerical results have been obtained for the nonlinear frequency amplitude dependence. Good agreement has been found between the nonlinear frequencies of the first mode from the present method and the published results discussed in reference [4].It will be noticed that the increase in the frequencies with increasing amplitude is more pronounced in the case of rectangular plates, and its less pronounced for skew plate with increasing skew angle $\theta$.

\section{References}

1. A.W. Leissa, Vibrations of plates, U.S Government printing office, NASA-SP160, pp. 1-360, (1969).

2. R. Benamar, M.M. Bennouna, and R.G. Whit, The effect of large vibration amplitudes on the mode shapes and natural frequencies of thin elastic structures, part II: fully clamped rectangular isotropic plates, Journal of sound and Vibration, vol. 164, pp. 295-316, (1992).

3. W. Han and M. Petyt, Geometrically nonlinear vibration analysis of thin, rectangular plates using the hierarchical finite element method-I: the fundamental mode of isotropic plates, Institute of Sound and Vibration Research, University of Southampton, vol.17, pp. 295-308, (1995).

4. G. Prathap and T. K. Varadan, Nonlinear flexural vibration of anisotropic skew plates, Department of Aeronautics, Indian Institute of Technology, (1978).

5. R.S. Alwar and N.R. Rao, Nonlinear analysis of orthotropic skew plates, AIAA Journal, vol. 11, pp. 495-498, (1973).

6. Chia, C.Y., Nonlinear analysis of plates, Mc-Graw Book Co., New York, (1980).

7. Y. Xiang, C.M. Wang and S. Kitipornchai, Buckling of skew Mindlin plates subjected to in-plane shear loadings, International Journal of Mechanical Sciences, vol. 37, No. 10, pp.1089-1101, (1995). 
8. M.K. Singha, R. Daripa, Nonlinear vibration of symmetrically laminated composite skew plates by finite element method, Department of Applied Mechanics, Indian Institute of Technology, Delhi, vol.42, pp.1144 - 1152 (2007). 